\title{
ATID - Authoring Tool for Instructional Design
}

\author{
Caio de Albuquerque Lucena Medeiros ${ }^{1}$, William Balbino Cunha Barbosa ${ }^{1}$, \\ Anderson Felinto ${ }^{2}$, Douglas Afonso Tenório de Menezes $^{2}$, Isabel Dillmann Nunes ${ }^{1}$, \\ Ulrich Schiel $^{2}$ \\ ${ }^{1}$ Faculdades de Ciências Sociais Aplicadas - FACISA \\ Campina Grande - PB - Brasil \\ ${ }^{2}$ Universidade Federal de Campina Grande - UFCG \\ Campina Grande - PB - Brasil \\ \{caiolucenamdr, anderson.fbarbosa, dougglas2002, \\ beldillnunes\}@gmail.com, williambalbinol4@ hotmail.com, \\ ulrich@computação.ufcg.edu.br
}

\begin{abstract}
Resumo. Este artigo apresenta a ferramenta ATID - Authoring Tool for Instructional Design. A ATID permite que um professor ou designer instrucional consiga modelar o sequenciamento das atividades de uma disciplina ou curso, considerando as condições para a transição entre elas. A notação utilizada pela ferramenta é a Rede de Atividades de Alto Nivel, a qual permite modelar atividades simples, sub redes, transições e repositórios. A notação está baseada em Redes de Petri, o que permite a verificação do modelo construído, identificando atividades que foram propostas mas que não necessitam ser finalizadas ou situações de deadlock, em que o aluno não consegue finalizar o curso.
\end{abstract}

\section{Cenário de Uso}

A construção de um ambiente de Educação On-line é composta pelas seguintes fases [Fetaji e Fetaji 2010]: planejamento, design instrucional, implementação, avaliação e análise. A fase de design instrucional - DI, por ser determinante na aplicação do conhecimento e por considerar as abordagens pedagógicas existentes, é importante no processo do ambiente de aprendizagem.

O planejamento do DI deve ser refletido, especialmente para a Educação On-line e em seus Ambientes Virtuais de Aprendizagem - AVA, em que há o uso de tecnologias, hipertextos e redes de comunicação interativa, sem limite de tempo ou lugar [Filatro 2010].

Os AVAs são responsáveis por disponibilizar conteúdos, meios de interação e atividades às pessoas que estão geograficamente distantes ou com indisponibilidade de horário comum. Porém, a maioria dos Ambientes Virtuais de Aprendizagem não proporcionam mecanismos de apoio ao Design Instrucional, sendo o processo de planejamento, construção e acompanhamento realizados de forma separados. Por isso, padrões como SCORM ${ }^{1}$ e IMS-LD ${ }^{2}$ são utilizados na tentativa de minimizar os problemas de DI [Vahldick et al. 2007; Filatro et al. 2006; Vantroys and Peter 2003].

\footnotetext{
${ }^{1}$ Sharable Content Object Reference Model
} 


\section{CBIE-LACLO 2015}

Anais dos Workshops do IV Congresso Brasileiro de Informática na Educação (CBIE 2015)

Tais padrões possuem a vantagem de permitir um reuso dos materiais construídos. O sequenciamento das atividades deve ser planejado antecipadamente e pode sofrer alterações durante a execução de um curso, porém os padrões não possuem uma forma de manter a integridade do sequenciamento no momento de uma alteração. Algumas questões devem ser realizadas durante o curso e não somente ao final, em sua avaliação. Questões como: Será que a alteração realizada mantém o objetivo do curso? Será que não "quebra" o sequenciamento criando situações em que atividades que eram essenciais tornam-se obsoletas? E o acompanhamento realizado no AVA, separa o ambiente de planejamento do ambiente de execução?

A utilização de Sistemas de Workflow aplicados a Educação trouxe um melhoramento em relação a tais questões. Possibilitando que tanto o planejamento como o acompanhamento sejam realizados de forma interativa. Porém, como mostra o trabalho de Spoelstra et al. (2008) é necessário criar mecanismos de flexibilidade, como ações atômicas que são pontos de mudanças permitidas.

Redes de Petri de Alto Nível podem ser utilizadas para suprir essa necessidade. Estas redes possuem uma base matemática e formal que permite o desenvolvimento e a simulação de sistemas [Padua et al. 2002]. Mais precisamente, Redes de Petri de Alto Nível, como as Redes de Petri Coloridas [Jensen 1997], permitem que atividades sejam modeladas, restrições de caminhos sejam especificadas e que as marcas sejam individualizadas, criando um ambiente propício para o planejamento (anterior ao curso) e acompanhamento de atividades realizadas por alunos (em tempo de execução) em ambientes virtuais de aprendizagem.

A Rede de Atividades de Alto Nível - RAAN é uma notação que traz as características necessárias para atender aos requisitos do planejamento de Design Instrucional e seu acompanhamento em tempo de execução. A RAAN utiliza como base formal a Rede de Projeto de Alto Nível - RPr, uma classe especial de Redes de Petri Colorida [Nunes e Schiel 2011]. A RPr garante as necessidades e características da RAAN e assim torna-se específica para o tipo de processo a ser modelado. Uma Rede de Projeto permite determinar a qualidade estrutural de uma Rede de Atividades. A qualidade estrutural é determinada pela análise do Grafo de Alcançabilidade correspondente [Nunes e Schiel 2013].

A definição da notação de Rede de Atividades de Alto Nível tem também o objetivo de tornar mais fácil e acessível aos usuários leigos o desenvolvimento e acompanhamento de Rede de Projetos de cursos. A RAAN permite especificar atividades e o estado de indivíduos e grupos em relação a essas atividades. Tais características são fundamentais em um processo de Design Instrucional. A partir da integração com um AVA, o modelo também pode acompanhar as atividades dos alunos em tempo de execução de um curso, permitindo assim identificar alunos em risco e pontos do DI mal planejados.

Foi especificada então uma ferramenta que implementa o processo proposto, denominada ATID - Authoring Tool for Instructional Design, a qual pode ser integrada

\footnotetext{
${ }^{2}$ Instructional Management System - Learning Design
} 
a um Ambiente Virtual de Aprendizagem que fornece informações dos alunos durante a execução do curso.

A ATID destina-se à professores e designers instrucionais pois tem o objetivo de permitir a construção do sequenciamento de atividades. Tal sequenciamento, utilizando como base uma matriz instrucional e suas características, deve ser aplicado de forma a ajudar no aprendizado.

\section{Desenvolvimento}

A ATID está sendo implementada utilizando a metodologia SCRUM e algumas práticas de XP, como a programação em pares. A Figura 3 mostra o Diagrama de Casos de Uso com as principais funcionalidades da ferramenta.

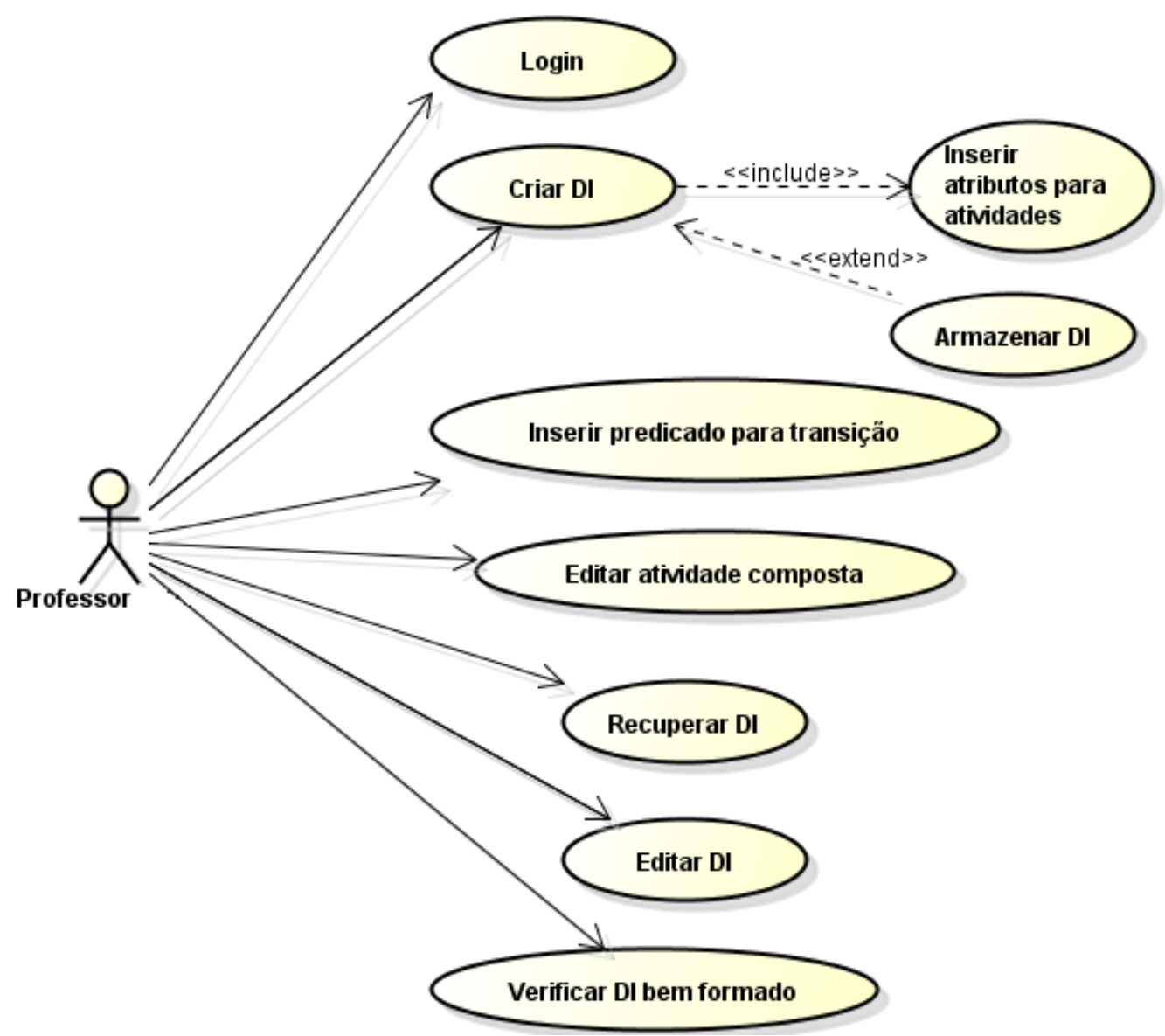

Figura 1. Diagrama de Casos de Uso da ATID

A ATID permite criar a estrutura do Design Instrucional de um determinado curso, definir ordens de prioridades, regras de validação para cada transição e definir prazos. Os DI's criados são armazenados afim de serem usados na execução do curso e, posteriormente, recuperados para reutilização ou como base para um novo modelo.

As condições de continuidade das atividades são definidas através das Condições de Guarda atribuídas às transições da Rede de Atividades. Tais condições ainda não 
foram implementadas pois é necessário o desenvolvimento de um analisador léxico e um analisador sintático para a gramática das condições descritas.

Um Design Instrucional criado deve ser analisado para que corresponda a uma Rede de Atividades bem formada. A ATID realiza essa verificação utilizando o Grafo de Alcançabilidade correspondente da RA. O Grafo de Alcançabilidade utilizado para verificação não é visualizado pelo usuário, sendo um recurso utilizado para identificar pontos de deadlock e evitar que seja possível chegar ao final da rede sem ter executado todas as atividades previstas para o processo.

A ferramenta está sendo desenvolvida em JAVA, utilizando seus recursos como Swing $^{3}$ e Graphics ${ }^{4}$. A ferramenta ainda está em fase de implementação e por isso ainda não foi possível realizar a sua validação.

\section{Apresentação do Software}

A ATID possui uma interface gráfica adequada para sua aplicação, em que o menu possui as opções de File e Edit como também específicas para construção da Rede de Atividades (Draw e Subnet) e para a verificação da rede em Algorithms, como mostra a Figura 2.

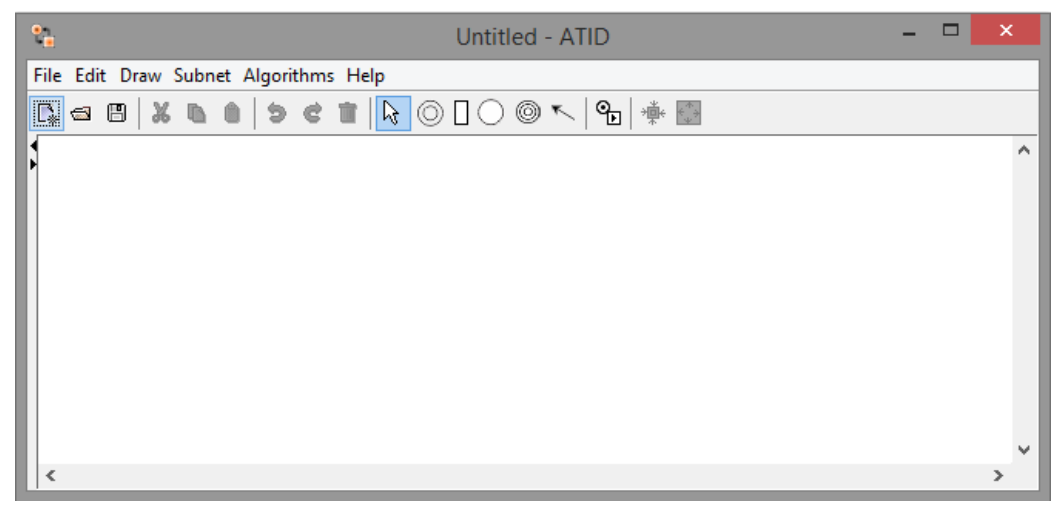

Figura 2. Interface gráfica da ATID

A construção da Rede de Atividades é realizada a partir da inserção dos seus elementos, conforme a necessidade do curso ou disciplina. A Figura 3 mostra uma rede de atividades formada com atividades simples (dois círculos) e composta (três círculos), transições (retângulo) e repositórios (círculo). Os repositórios são elementos que indicam o armazenamento de documentos, tanto para serem consumidos pelos alunos ou produzidos por eles em alguma atividade. O DI começa com a atividade begin e atividade $\boldsymbol{e n d}$, tais atividades não possuem atributos (a não ser o próprio nome) e representam o início e fim do DI respectivamente.

A correta associação entre os elementos também é tratada na ATID. A Figura 4 mostra a tentativa de associar uma atividade em outra, o que não é permitido devido ao conceito de Redes de Petri utilizado, e a inibição por parte da ferramenta.

\footnotetext{
${ }^{3}$ http://docs.oracle.com/javase/6/docs/technotes/guides/swing/

${ }^{4}$ http://docs.oracle.com/javase/7/docs/api/java/awt/Graphics.html
} 


\section{CBIE-LACLO 2015}

Anais dos Workshops do IV Congresso Brasileiro de Informática na Educação (CBIE 2015)

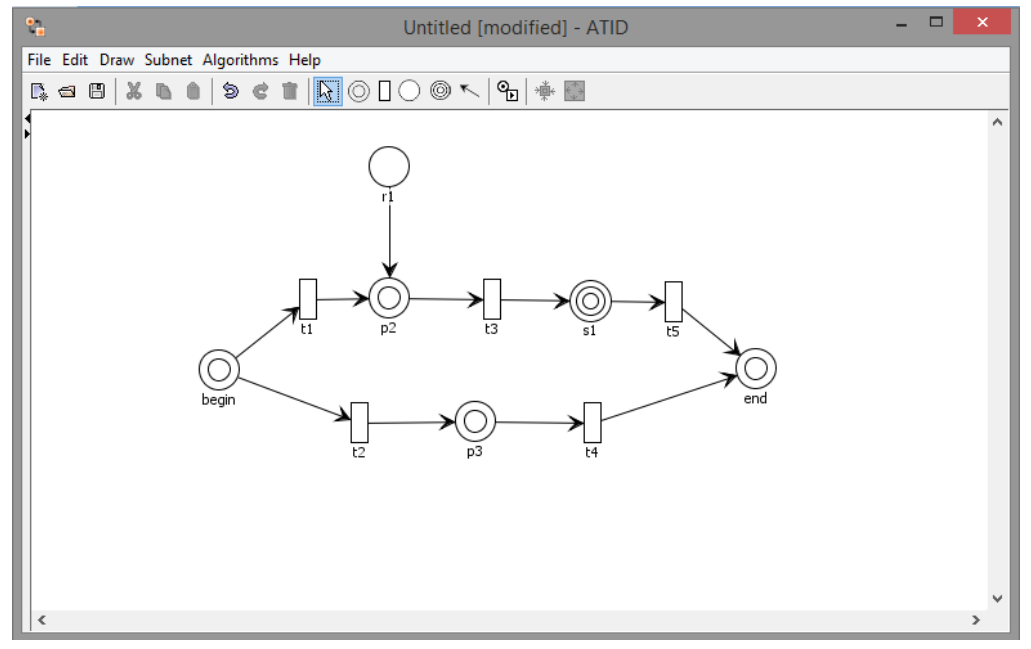

Figura 3. Rede de Atividades na ATID

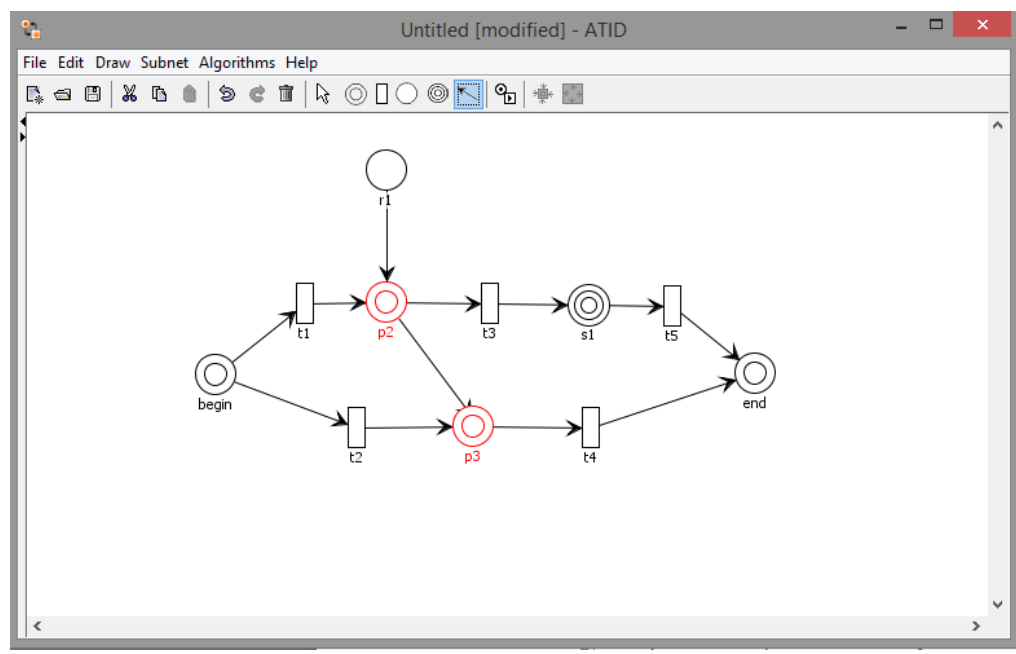

Figura 4. Erro ao ligar dois elementos iguais (atividades simples).

A fase de análise da Rede de Atividades para identificar se a rede está bem formada, por meio do Grafo de Alcançabilidade, deve ser inicializada pela definição de uma marca na atividade inicial e a execução das transições que estão disponíveis para serem disparadas. Caso a rede tenha um deadlock ou que permita uma atividade não ser finalizada para o encerramento do curso, a ATID indicará, respectivamente, com apenas transições em vermelho (Figura 5) e com uma marca na atividade que não necessitou ser finalizada e outra na atividade end (Figura 6).

O código fonte da ATID ainda não está disponível devido ao estágio inicial de desenvolvimento da ferramenta. Releases serão disponibilizadas assim que estiverem funcionais, sob a licença GNU GPL. 
CBIE-LACLO 2015

Anais dos Workshops do IV Congresso Brasileiro de Informática na Educação (CBIE 2015)

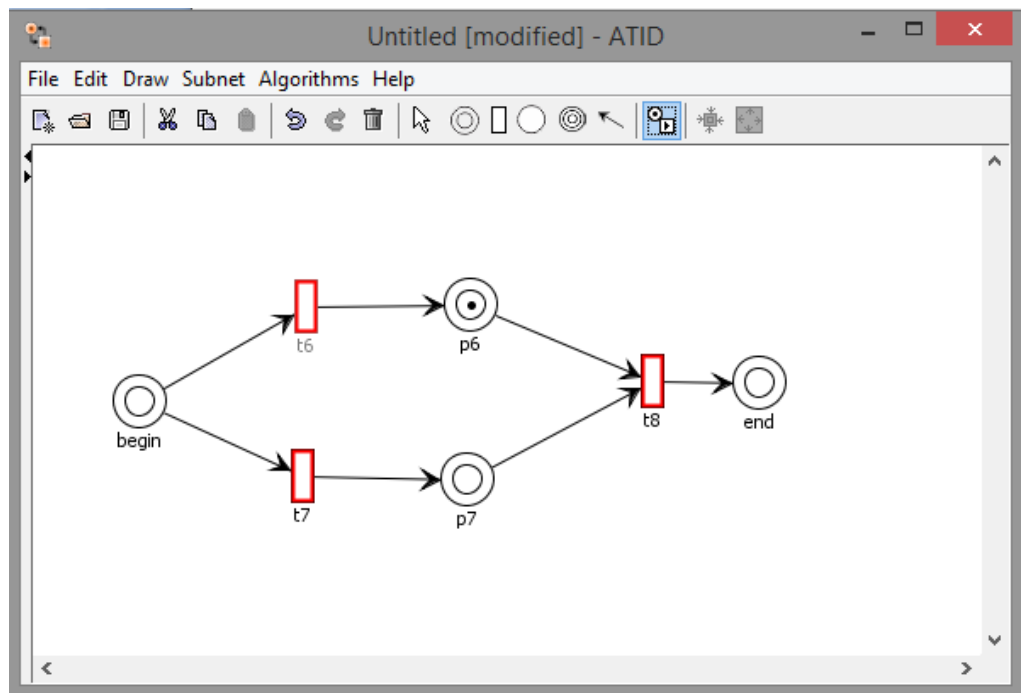

Figura 5. Rede de Atividades com deadlock.

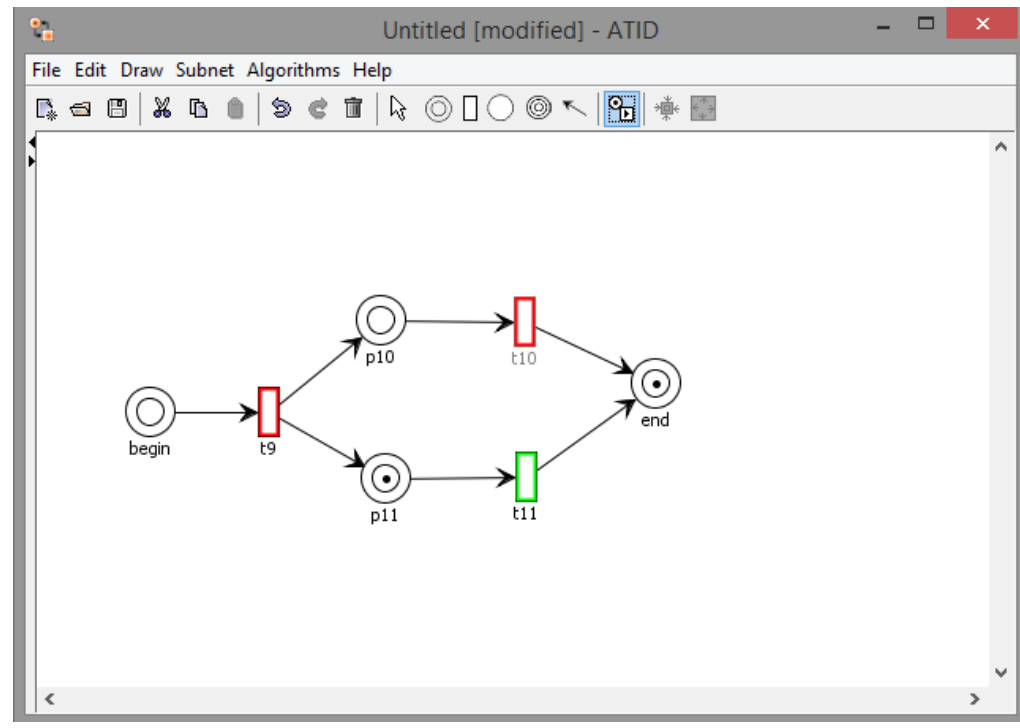

Figura 6. Rede de Atividades com atividades não finalizadas.

\section{Considerações Finais}

A ferramenta ATID permite a construção de Designs Instrucionais por meio da notação de Rede de Atividades de Alto Nível.

Atualmente, outras funcionalidades estão sendo implementadas:

- Novo elemento chamado Evento, o qual permite que eventos externos tais como a utilização de recursos sejam considerados;

- Condições para as transições, fazendo com que o professor possa modelar caminhos alternativos para os alunos conforme sua frequência, notas ou performance. 


\section{CBIE-LACLO 2015}

Anais dos Workshops do IV Congresso Brasileiro de Informática na Educação (CBIE 2015)

A partir da ferramenta finalizada será possível intregá-la aos diversos AVAs existentes. A proposta inicial é implantar a sua integração com o Moodle, para que a ferarmenta seja, não somente um editor, mas também uma interface gráfica para o acompanhamento das atividades realizadas no ambiente virtual.

A ATID possui a característica de ser independente de AVA, assim, para sua integração é necessário que aplicações de integração sejam implementadas sem que tenha que alterar a própria ferramenta ou o ambiente virtual de aprendizagem. Essa característica de diversidade torna-se relevante para considerá-la uma aplicação comerciável, pois pode atender a diferentes AVAs.

\section{Referências}

Fetaji, B; Fetaji, M.(2010) E-Learning Indicators: A Multidimensional Model for Planning Developing and Evaluating E-Learning Software Solutions. In: E-Learning, experiences and future. Edited by Safeeullah Soomro and published by In-Teh. Pag 1- 34. 2010

Filatro, A.; Boaretto, R.; Nunes, C. (2006) Learning Activities Editor Tool. TDIA - III Workshop. Pág. 152-154.

Filatro, A. (2010) Design Instrucional Contextualizado - educação e tecnologia. $3^{\text {a }}$ edição. Editora Senac - São Paulo. 215 páginas.

Jensen, K. (1997) Coloured Petri Nets - Basic Concepts, Analysis Methods and Practical Use. Volume 1. Second Edition. Springer-Verlag Berlin Heidelberg New York.

Nunes, I. D.; Schiel, U.(2011) Design Instrucional e seu acompanhamento em tempo real utilizando Rede de Atividades. In: Simpósio Brasileiro de Informática na Educação, 2011, Aracajú. Simpósio Brasileiro de Informática na Educação.

Nunes, I. D. ; Schiel, U.(2013) Utilização de Grafo de Alcançabilidade para a verificação de Design Instrucional bem formado. In: Desafie! (CSBC), 2013, Maceió. Desafie! (CSBC).

Pádua, S. I. D. de; Silva, A. R. Y. da; Inamasu, R. Y.; Porto, A. J. V. (2002) Redes de Petri aplicadas aos Sistemas de Gerenciamento de Workflow. XXII Encontro Nacional de Engenharia de Produção. Curitiba - PR. 2002.

Spoelstra, H.; Matera, M.; Rusman, E.; Bruggen, J. van.; Koper, R. (2008) Bridging the gap between instructional design and double loop learning. International Journal of Web-Based Learning and Teaching Technologies (IJWLTT). Volume 3, Issue1. 12 pp.

Vahldick A.; Santiago, R. de; Raabe, A. L. A. (2007) Aplicação das Técnicas de Projeto Instrucional 4C/ID na Produção de Objetos de Aprendizagem em Conformidade com o SCORM usando um Software Livre como Ferramenta de Autoria. Revista RENOTE - Novas Tecnologias na Educação. V. 5, nº 2. 2007.

Vantroys, T. and Peter, Y. (2003) COW, a Flexible Plataform for the Enactment of Learning Scenarios. Lecture Notes in Computer Science. 